\title{
Effect of intravenous infusion of rocuronium on emergence from propofol anesthesia in rats
}

\section{Kaoru Suzuki}

Jikei University School of Medicine

Hiroshi Sunaga ( $\sim$ hs-031@jikei.ac.jp )

Jikei University School of Medicine

Kentaro Yamakawa

Jikei University School of Medicine

Yoshifumi Suga

Jikei University School of Medicine

Ichiro Kondo

Jikei University School of Medicine

Tsunehisa Tsubokawa

Jikei University School of Medicine

Shoichi Uezono

Jikei University School of Medicine

\section{Research article}

Keywords: Emergence, Propofol, Rat, Rocuronium, Sugammadex

Posted Date: February 11th, 2020

DOI: https://doi.org/10.21203/rs.2.23130/v1

License: (c) (i) This work is licensed under a Creative Commons Attribution 4.0 International License.

Read Full License 


\section{Abstract}

Background: Central nervous system effects of neuromuscular blocking agents have been indicated in some case reports. We investigated whether intravenous (IV) infusion of rocuronium affects emergence from propofol anesthesia in rats.

Methods: We used Sprague Dawley rats. Propofol infusion was initiated with a bolus of $15 \mathrm{mg} / \mathrm{kg}$ and continued at a rate of $40 \mathrm{mg} / \mathrm{kg} / \mathrm{h}$. For the rocuronium group $(\mathrm{n}=18)$, rocuronium was administered as an initial IV bolus of $5 \mathrm{mg} / \mathrm{kg}$ followed by continuous infusion at a rate of 250,500 , or $1000 \mu \mathrm{g} / \mathrm{kg} / \mathrm{min}$ along with propofol infusion. Infusion was continued for $60 \mathrm{~min}$, and sugammadex ( $32 \mathrm{mg} / \mathrm{kg}$ ) was injected at the end of infusion. In a separate group of rats $(n=12)$, normal saline was administered along with propofol infusion. After continuous infusion for $60 \mathrm{~min}$, normal saline or sugammadex $(32 \mathrm{mg} / \mathrm{kg})$ was injected. The time to emergence from propofol anesthesia was evaluated. To ascertain possible factors affecting emergence, the neuromuscular blocking, circulatory, and respiratory properties of IV rocuronium infusion at $1000 \mu \mathrm{g} / \mathrm{kg} / \mathrm{min}$ were assessed $(n=18)$.

Results: The time to emergence from propofol anesthesia was $239 \pm 94 \mathrm{~s}$ after simultaneous infusion of normal saline without rocuronium and was $346 \pm 78,518 \pm 134$, and $638 \pm 219$ s after IV infusion of rocuronium at 250,500 , and $1000 \mu \mathrm{g} / \mathrm{kg} / \mathrm{min}$, respectively. The simultaneous IV infusion of rocuronium dose-dependently increased the time to emergence $(\rho=0.624 ; p=0.006)$. Sugammadex alone did not delay emergence $(280 \pm 60 \mathrm{~s} ; \mathrm{p}=0.39)$. Neuromuscular blockade induced by rocuronium at 1000 $\mu \mathrm{g} / \mathrm{kg} / \mathrm{min}$ was completely antagonized at $99 \pm 21 \mathrm{~s}$ by sugammadex $(32 \mathrm{mg} / \mathrm{kg}$ ). Mean arterial pressure, heart rate, partial pressures of oxygen and carbon dioxide, and $\mathrm{pH}$ were not affected by rocuronium infusion.

Conclusions: Our results show that IV infusion of rocuronium delays the emergence from propofol anesthesia in rats, despite the complete recovery from neuromuscular blockade by sugammadex. The use of neuromuscular blocking agents in neonates or patients with cerebrovascular diseases, whose blood-brain barrier might be immature or disrupted, should be carefully considered.

\section{Background}

The major effect of neuromuscular blocking agents (NMBAs) is muscle paralysis induced via the prevention of neuromuscular transmission at the neuromuscular junction. The effect of NMBAs on the central nervous system (CNS) is a topic of investigation. We reported that pancuronium injected into the lateral ventricle in rats dose-dependently enhanced the depth of isoflurane anesthesia [1]. Our results suggested that the mechanism might be inhibition of neuronal nicotinic acetylcholine receptor transmission in the cerebrum. Another study in nonanesthetized rats showed that the administration of NMBAs into the lateral ventricle exerted a variety of actions, such as sedation and seizure, according to the dose [2]. These findings indicate that NMBAs have some effects on the CNS. However, when NMBAs 
are administered intravenously in clinical practice, these effects are generally considered to be irrelevant because the blood-brain barrier (BBB) prevents NMBAs from entering the cerebrum.

The BBB strictly controls the movement of substances between blood vessels and the cerebral parenchyma. Neuromuscular blocking agents, such as pancuronium, vecuronium, and rocuronium, are quaternary ammonium compounds of high molecular weight; hence, NMBAs generally cannot pass the BBB. However, in neonates or patients with certain clinical conditions (e.g., cerebrovascular diseases), whose BBB may be immature or disrupted, NMBAs can enter the cerebrum. For example, rocuronium was detected in the cerebrospinal fluid (CSF) after intravenous (IV) administration of rocuronium in patients undergoing cerebral aneurysm clipping [3], and the passage of rocuronium from blood vessels to the cerebrum has been suggested in neonates $[4,5]$.

Previous studies suggest that NMBAs act at several locations in the cerebrum and have a variety of effects when administered directly to the CSF or CNS $[1,2,6,7]$. The effects of NMBAs when passed across the BBB after IV administration remain unclear. We hypothesized that it might be possible to investigate this issue in an animal model because species-specific differences with regard to the BBB might result in increased permeability of NMBAs in some animals compared to humans [8]. We hypothesized that NMBAs can enter the cerebrum in rats after IV infusion and that we might be able to recognize the effect on the CNS during emergence from general anesthesia. Such findings might contribute to the accurate neurologic assessment after or improved safety of general anesthesia with NMBAs in patients in whom the BBB may be immature or disrupted. In the present study, we evaluated the effect of IV infusion of rocuronium on the time to emergence from propofol anesthesia in rats. In addition, we assessed the neuromuscular blocking, circulatory, and respiratory properties of rocuronium infusion to exclude other possible factors affecting emergence.

\section{Methods}

Male Sprague Dawley rats (Japan SLC, Shizuoka, Japan) weighing $294 \pm 10 \mathrm{~g}$, aged 9 to 10 weeks, were used (total $n=48$ : $n$ refers to number of animals). Rats were housed with a 12 hours light-dark cycle with lights on at 7 am in a temperature $\left(22 \pm 2^{\circ} \mathrm{C}\right)$ and humidity $(55 \pm 10 \%)$ controlled room and allowed free access to water and food in a polymethylpentene cage filled with paper bedding (PaperClean ${ }^{\mathrm{TM}}$, Japan SLC). All protocols were approved by the Institutional Animal Care and Use Committee of The Jikei University, Tokyo, Japan (approval number 2016-023). Animal care and experiments conformed to the Fundamental Guidelines for Proper Conduct of Animal Experiment and Related Activities in Academic Research Institutions by the Ministry of Education, Culture, Sports, Science and Technology of Japan.

\section{Reagents}

Powdered rocuronium bromide was purchased from Tokyo Chemical Industry (Tokyo, Japan) and serially diluted in normal saline. Clinical formulations of propofol (Maruishi Pharmaceutical, Osaka, Japan) and sugammadex (MSD, Tokyo, Japan) were used. 


\section{Experimental Setup}

Rats were anesthetized with isoflurane. After induction of anesthesia at $4-5 \%$, isoflurane was maintained at $2-3 \%$. The surgical procedure was initiated after confirming no response to foot pinch. Lidocaine was infiltrated into the incision sites. Respiratory rate and response to foot pinch were monitored during surgery, and the concentration of isoflurane was increased when an increase in respiratory rate or a withdrawal response to foot pinch was observed. The trachea was cannulated with a 16-g catheter, and the rat was ventilated at $10 \mathrm{ml} / \mathrm{kg}$ and 60 breaths/min with isoflurane and oxygen with a rodent ventilator (model 683; Harvard Apparatus, Holliston, MA, USA). A heating device was used to maintain body temperature at $36^{\circ} \mathrm{C}$ to $37^{\circ} \mathrm{C}$. For the emergence, circulation, and respiration experiments, the right femoral vein was cannulated with a 24-g catheter for drug administration. For the neuromuscular blockade (NMB) experiment, the right jugular vein was cannulated for drug administration to keep the lower limb intact for neuromuscular monitoring.

\section{Assessment of the effect of rocuronium on time to emergence}

Rats were randomly allocated to the rocuronium- or normal saline-infusion group. After completion of the experimental setup, isoflurane was discontinued. When the concentration of isoflurane decreased to $1 \%$, propofol infusion was initiated with a bolus dose of $15 \mathrm{mg} / \mathrm{kg}$ and continued at a rate of $40 \mathrm{mg} / \mathrm{kg} / \mathrm{h}$. For the rocuronium group $(n=18)$, rocuronium was administered as an initial IV bolus of $5 \mathrm{mg} / \mathrm{kg}$ followed by continuous infusion at a rate of 250,500 , or $1000 \mu \mathrm{g} / \mathrm{kg} / \mathrm{min}$ along with the propofol infusion for $60 \mathrm{~min}$. At the discontinuation of infusion, sugammadex $(32 \mathrm{mg} / \mathrm{kg})$ was injected, and the line was flushed with $0.5-\mathrm{ml}$ normal saline. In a separate group of rats, normal saline was administered as a continuous infusion at a rate of $1.5 \mathrm{ml} / \mathrm{kg} / \mathrm{h}$ (in the same volume as the rocuronium group) along with propofol infusion for $60 \mathrm{~min}$. At the end of infusion, $0.1-\mathrm{ml}$ normal saline $(\mathrm{n}=6)$ or sugammadex $(32 \mathrm{mg} / \mathrm{kg} ; \mathrm{n}=6)$ was injected, and the line was flushed with $0.5-\mathrm{ml}$ normal saline. In both groups, the vibrissae were pulled at intervals of $30 \mathrm{~s}$ until a sign of emergence (movement of tongue, mouth, or limbs) was observed. The time to emergence from propofol anesthesia, defined as the time from flush of the IV line at the termination of infusion to the appearance of a sign of emergence, was assessed.

\section{Assessment of neuromuscular blocking, circulatory, and respiratory properties of rocuronium}

After completion of the experimental setup, the neuromuscular blocking, circulatory, and respiratory properties of rocuronium infusion were assessed in separate groups of rats. For the NMB experiment $(n=$ 6), the sciatic nerve was exposed to attach a small slide electrode, and the tendon of the gastrocnemius muscle was connected to a force transducer (MLTF500/ST; ADInstruments, Colorado Springs, CO, USA). The sciatic nerve was stimulated at $0.1 \mathrm{~Hz}$ with a supramaximal stimulation of 0.2 -ms pulse width using 
an electric stimulator (SEN-3401; Nihon Kohden, Tokyo, Japan) and an isolator (SS-104J; Nihon Kohden) to elicit twitch responses of the gastrocnemius muscle. Single twitch responses were amplified and recorded using PowerLab (ADInstruments). After establishment of the magnitude of the twitch responses, isoflurane was discontinued. When the concentration of isoflurane decreased to $1 \%$, propofol infusion was initiated with a bolus dose of $15 \mathrm{mg} / \mathrm{kg}$ and continued at a rate of $40 \mathrm{mg} / \mathrm{kg} / \mathrm{h}$. Rocuronium was administered as an initial IV bolus of $5 \mathrm{mg} / \mathrm{kg}$ followed by continuous infusion at a rate of $1000 \mu \mathrm{g} / \mathrm{kg} / \mathrm{min}$, which was the greatest infusion rate in the emergence experiment, along with propofol infusion for $60 \mathrm{~min}$. At the end of infusion, sugammadex $(32 \mathrm{mg} / \mathrm{kg}$ ) was injected, and the line was flushed with $0.5-\mathrm{ml}$ normal saline. Both NMB and antagonism were assessed, and the time from injection of sugammadex to complete recovery of twitch height was determined.

For the assessment of circulatory and respiratory effects, rats were randomly allocated to the normal saline- or rocuronium-infusion group. The carotid artery was cannulated with a 24-g catheter and connected to a pressure transducer. Arterial pressure and heart rate (HR) were recorded throughout the experiment with PowerLab. Propofol infusion was performed in the same manner as that for the emergence experiment. Infusion of normal saline at $1.5 \mathrm{ml} / \mathrm{kg} / \mathrm{h}$ (normal saline group; $\mathrm{n}=6$ ) or rocuronium at $1000 \mu \mathrm{g} / \mathrm{kg} / \mathrm{min}$ after an initial bolus of $5 \mathrm{mg} / \mathrm{kg}$ (rocuronium group; $\mathrm{n}=6$ ) was performed along with propofol infusion for $60 \mathrm{~min}$. At the discontinuation of infusion, the line was flushed with 0.6$\mathrm{ml}$ normal saline for the normal saline group or $32-\mathrm{mg} / \mathrm{kg}$ sugammadex followed by $0.5-\mathrm{ml}$ normal saline for the rocuronium group. Blood samples were collected for blood gas analysis at the following time points: before infusion; at 30 min after initiation of infusion; and at 3 min after flushing the line at the discontinuation of infusion.

All experiments were conducted in a laboratory during the light phase ( $7 \mathrm{am}$ to $7 \mathrm{pm})$. Each rat was studied once and sacrificed by intravenous pentobarbital overdose after the experiment.

\section{Data analysis}

The primary outcome was the relation between time to emergence from propofol anesthesia in rats and infusion rate of rocuronium along with propofol. The Spearman rank correlation coefficient ( $\rho$ ) was used to analyze the relation between time to emergence and infusion rate of rocuronium. We estimated that the correlation coefficient would be approximately 0.65 , and to achieve a power of $80 \%$ and a type 1 error of $0.05,16$ samples were required. Statistical comparison was made by unpaired two-tailed Student $t$ test to ascertain whether sugammadex alone was related to time to emergence from propofol anesthesia. Mean arterial pressure (MAP) and HR at bolus injection of propofol and rocuronium (time 0 ) and at 1, 3, 5, $10,20,30,45$, and 60 min after initiating rocuronium infusion with propofol, as well as the partial pressures of oxygen $\left(\mathrm{PaO}_{2}\right)$ and carbon dioxide $\left(\mathrm{PaCO}_{2}\right)$ and $\mathrm{pH}$ before infusion, at $30 \mathrm{~min}$ after initiating infusion, and at 3 min after flushing the line at the discontinuation of infusion, were compared to those in response to normal saline administration with propofol using two-way repeated measures analysis of variance with a post hoc Student $t$ test and Bonferroni correction for multiple comparisons. SigmaPlot 13 
(Systat Software, Inc., San Jose, CA, USA) was used for analysis. All values are presented as mean \pm standard deviation. For all statistical comparisons, a value of $p<0.05$ was considered significant.

\section{Results}

The time to emergence from propofol anesthesia was $239 \pm 94 \mathrm{~s}$ after simultaneous infusion of normal saline without rocuronium. When rocuronium was administered at a rate of 250,500 , and $1000 \mu \mathrm{g} / \mathrm{kg} / \mathrm{min}$ along with propofol, the time to emergence was $346 \pm 78,518 \pm 134$, and $638 \pm 219 \mathrm{~s}$, respectively. The relation between the time to emergence from propofol anesthesia and the infusion rate of rocuronium is shown in Fig. 1. The Spearman rank correlation coefficient suggested that the time to emergence from propofol anesthesia was dose-dependently prolonged by simultaneous infusion of rocuronium $(\rho=0.624 ; p=0.006)$. Sugammadex alone did not affect the time to emergence from propofol anesthesia $(280 \pm 60 s ; p=0.39)$.

Muscle twitch was completely blocked throughout rocuronium infusion at a rate of $1000 \mu \mathrm{g} / \mathrm{kg} / \mathrm{min}$ in all rats and was completely recovered at $99 \pm 21 \mathrm{~s}$ by $32 \mathrm{mg} / \mathrm{kg}$ sugammadex.

Values for MAP and HR during rocuronium or normal saline infusion with propofol are shown in Fig. 2. Values for MAP and HR showed significant differences between time points in each group; however, those during rocuronium infusion did not differ significantly from those during normal saline infusion at any time point.

Values for $\mathrm{PaO}_{2}, \mathrm{PaCO}_{2}$, and $\mathrm{pH}$ before infusion, at $30 \mathrm{~min}$ after initiating infusion, and at $3 \mathrm{~min}$ after flushing the line at the discontinuation of infusion are shown in Fig. 3. Values for $\mathrm{PaO}_{2}$ showed a significant difference between time points in the normal saline group, and those for $\mathrm{PaCO}_{2}$ showed significant differences between time points in each group; however, values for $\mathrm{PaO}_{2}, \mathrm{PaCO}_{2}$, and $\mathrm{pH}$ did not differ significantly between the rocuronium group and the normal saline group at any time point.

\section{Discussion}

The results of the present study show that continuous IV infusion of rocuronium dose-dependently delayed the emergence from propofol anesthesia in rats. The definitive mechanism was not elucidated. Nonetheless, sugammadex rapidly reversed rocuronium-induced NMB; therefore, the rats were not immobilized despite potentially being aware. In addition, sugammadex alone did not affect the time to emergence. The effect of propofol infusion with rocuronium on circulation and respiration did not differ from propofol infusion without rocuronium; therefore, low cerebral blood flow was not a contributing factor.

Neuromuscular blocking agents induce mainly muscle paralysis, owing to the prevention of neuromuscular transmission at the neuromuscular junction, and potentially some effects via other sites of action. Rocuronium might exhibit a vagal blocking effect at clinically relevant doses and a 
bronchoconstriction effect at high doses by interacting with muscarinic receptors $[9,10]$. In addition, results of an animal study showed that IV administration of pancuronium enhanced the effect of isoflurane on the cerebrum [11]. The complex effects of and mechanisms underlying IV administration of NMBAs on the CNS remain unclear. The present study suggests that continuous IV infusion of rocuronium has a side effect of delaying the emergence from propofol anesthesia in rats.

One possible mechanism for the rocuronium-induced delay in rats might be a direct effect on the CNS by entering the cerebrum. In general, NMBAs cannot pass the BBB in humans because they are large quaternary ammonium compounds. However, a recent study showed species-specific differences in the BBB between rodents and primates [8]. Unidentified transporters might exist in rats, or the permeability of rocuronium might differ from that in humans; therefore, rocuronium might pass the BBB and enter the cerebrum in rats. In addition, the dosage for continuous infusion of NMBAs required to maintain a consistent level of NMB varies among species. Whereas the infusion rate of rocuronium to obtain twitch depression of $90-95 \%$ (90\%-95\% effective dose $\left.\left[\mathrm{ED}_{90}, \mathrm{ED}_{95}\right]\right)$ in humans is reported to be $9 \mu \mathrm{g} / \mathrm{kg} / \mathrm{min}$, the $E D_{90}$ for $N M B$ with continuous infusion of rocuronium in rats is $119 \mu \mathrm{g} / \mathrm{kg} / \mathrm{min}[12,13]$. The infusion rates in the present study, 250,500, and $1000 \mu \mathrm{g} / \mathrm{kg} / \mathrm{min}$, were equal to approximately 2,4 , and 8 times the $\mathrm{ED}_{90}$ for $\mathrm{NMB}$, respectively; therefore, they might not be excessive doses for this animal model. Nevertheless, access to the cerebrum might have been facilitated by the large number of molecules administered during infusion.

It is unknown what the effects of NMBAs administered via IV infusion might be if they should pass across the BBB. The effects of NMBAs administered directly to the CSF or brain slices have been reported $[1,2,6,7]$, and the results imply that NMBAs act on several locations in the cerebrum and have a variety of effects. We previously reported that microinjection of pancuronium into the lateral ventricle at doses of $1.6-16 \%$ of the $E D_{50}$ for NMB in rats dose-dependently enhanced the depth of isoflurane anesthesia [1]. Another study reported pancuronium-induced seizures when a dose of 2.3 times the $\mathrm{ED}_{50}$ for NMB was injected into the lateral ventricle in rats [2]. These findings suggest that pancuronium might have an excitatory effect at high concentrations and an inhibitory effect at low concentrations in the area of the lateral ventricle. Rocuronium might have similar effects because it is an analog of pancuronium. If seizures had occurred in the present study, the rats could not move because of complete NMB during IV infusion. The concentration of rocuronium in the CSF was not measured in the present study. Thus, it is difficult to speculate as to whether rocuronium showed an excitatory or inhibitory effect on the cerebrum. However, because either effect could result in delayed emergence from general anesthesia, the direct action of rocuronium on the CNS could be considered a probable reason.

Another possible mechanism might be an indirect effect of rocuronium on the CNS. One study suggested indirect inhibition of cerebral stimulation by pancuronium as a result of a decrease in muscle afferent activity [14]. This mechanism might apply to the CNS effect during rocuronium infusion in the present study; however, after recovery from NMB, it might not. Because the muscles of the limbs are more sensitive to the effects of NMBAs than other muscles, which explains the slower recovery from NMB [15], 
the muscle tone of almost all skeletal muscles should have been recovered from NMB at the time of complete reversal of NMB of the gastrocnemius muscle. Hence, the decrease in muscle afferent activity should have been wearing off at the same time. An indirect action on the CNS would not be expected to affect the time to emergence.

Circulatory effects might cause delayed emergence from propofol anesthesia because they might alter not only cerebral blood flow but also the plasma concentration of propofol [16]. The $\mathrm{PaCO}_{2}$ and $\mathrm{pH}$ might affect cerebral blood flow as well. Compared with normal saline infusion with propofol, rocuronium infusion with propofol did not elicit significant differences in $\mathrm{MAP}, \mathrm{HR}, \mathrm{PaCO}_{2}, \mathrm{PaO}_{2}$, or pH at any time point in the present study; therefore, circulatory and respiratory effects on the emergence from propofol anesthesia should be excluded.

Sugammadex did not antagonize the effect of rocuronium on emergence as fast as that on NMB in the present study. Sugammadex has a molar mass of $2178 \mathrm{~g} / \mathrm{mol}$, which is approximately 3.5 times greater than the molar mass of rocuronium. Hence, sugammadex generally binds rocuronium in the blood vessels and cannot move outside the blood vessels. According to the concentration gradient, rocuronium moves to the blood vessels from the distributed tissue. The slower recovery of awareness during emergence in the present study might be attributed to the slow efflux of rocuronium from the cerebrum or high sensitivity to rocuronium in the CNS. A case report showed that both peripheral and presumed CNS effects of rocuronium disappeared immediately after the administration of sugammadex in a neonate [5]. If sugammadex were not administered in the present study, recovery from muscle paralysis as well as recovery of awareness during emergence from propofol anesthesia would have been much longer. Sugammadex might be useful to rapidly reverse the effect of rocuronium on emergence as well as on NMB.

In general, the time to emergence should be defined as the time to return of the righting reflex $[17,18]$. However, because the return of the righting reflex appears after forelimb movement or mastication in experimental animals during the emergence from anesthesia [19], that definition is inadequate for more invasive studies in which tracheotomy and mechanical ventilation are required. Hence, the time to emergence from propofol anesthesia was defined as the time from flush of the IV line at the termination of infusion to the appearance of movement of the tongue, mouth, or limbs in the present study.

\section{Conclusions}

The present study shows that continuous IV infusion of rocuronium delays the emergence from propofol anesthesia in rats. This is consistent with the signs described in case reports and findings of previous studies. Studies to detect rocuronium in the CSF or CNS and electrophysiologic studies will be required to obtain conclusive evidence regarding the mechanism. Nonetheless, the use of NMBAs in neonates or patients with cerebrovascular diseases, whose BBB might be immature or disrupted, should be carefully considered. 


\section{Abbreviations}

BBB: Blood-brain barrier; CNS: Central nervous system; CSF: Cerebrospinal fluid; HR: Heart rate; IV: Intravenous; MAP: Mean arterial pressure; NMB: Neuromuscular blockade; NMBAs: Neuromuscular blocking agents; PaCO2: Partial pressure of carbon dioxide; PaO2: Partial pressure of oxygen.

\section{Declarations}

\section{Ethics approval and consent to participate}

All protocols were approved by the Institutional Animal Care and Use Committee of The Jikei University, Tokyo, Japan (approval number 2016-023). Animal care and experiments conformed to the Fundamental Guidelines for Proper Conduct of Animal Experiment and Related Activities in Academic Research Institutions by the Ministry of Education, Culture, Sports, Science and Technology of Japan.

\section{Consent for publication}

Not applicable

\section{Availability of data and materials}

The datasets used and/or analyzed during the current study are available from the corresponding author on reasonable request.

\section{Competing interests}

All authors declare that they have no competing interests.

\section{Funding}

This research was supported by The Jikei University Research Fund and JSPS KAKENHI grant number JP18K08832. The funding bodies had no role in the design of the study and collection, analysis, and interpretation of data and in writing the manuscript.

\section{Authors' contributions}

$\mathrm{KS}, \mathrm{HS}, \mathrm{TT}$, and SU designed the study. KS, HS, and IK collected the data. HS, KY, YS, and TT analyzed the data. HS wrote the initial draft of the manuscript. KS, KY, YS, IK, TT, and SU revised the manuscript. All authors read and approved the final manuscript.

\section{Acknowledgements}

Not applicable

\section{References}


1. Miyazaki $Y$, Sunaga $H$, Hobo S, Miyano $K$, Uezono S. Pancuronium enhances isoflurane anesthesia in rats via inhibition of cerebral nicotinic acetylcholine receptors. J Anesth. 2016;30(4):671-6.

2. Szenohradszky J, Trevor AJ, Bickler P, Caldwell JE, Sharma ML, Rampil IJ, et al. Central nervous system effects of intrathecal muscle relaxants in rats. Anesth Analg. 1993;76(6):1304-9.

3. Fuchs-Buder T, Strowitzki M, Rentsch K, Schreiber JU, Philipp-Osterman S, Kleinschmidt S. Concentration of rocuronium in cerebrospinal fluid of patients undergoing cerebral aneurysm clipping. Br J Anaesth. 2004;92(3):419-21.

4. Joyce C, Greenwald BM, Han P. Bilateral dilated nonreactive pupils in a neonate after surgery. A A Case Rep. 2016;6(9):286-7.

5. Langley RJ, McFadzean J, McCormack J. The presumed central nervous system effects of rocuronium in a neonate and its reversal with sugammadex. Paediatr Anaesth. 2016;26(1):109-11.

6. Cardone C, Szenohradszky J, Yost S, Bickler PE. Activation of brain acetylcholine receptors by neuromuscular blocking drugs. A possible mechanism of neurotoxicity. Anesthesiology. 1994;80(5):1155-61.

7. Chiodini FC, Tassonyi E, Fuchs-Buder T, Fathi M, Bertrand D, Muller D. Effects of neuromuscular blocking agents on excitatory transmission and gamma-aminobutyric acidA-mediated inhibition in the rat hippocampal slice. Anesthesiology. 1998;88(4):1003-13.

8. Hoshi Y, Uchida Y, Tachikawa M, Inoue T, Ohtsuki S, Terasaki T. Quantitative atlas of blood-brain barrier transporters, receptors, and tight junction proteins in rats and common marmoset. J Pharm Sci. 2013;102(9):3343-55.

9. Stevens JB, Hecker RB, Talbot JC, Walker SC. The haemodynamic effects of rocuronium and vecuronium are different under balanced anaesthesia. Acta Anaesthesiol Scand. 1997;41(4):502-5.

10. Jooste E, Zhang Y, Emala CW. Neuromuscular blocking agents' differential bronchoconstrictive potential in Guinea pig airways. Anesthesiology. 2007;106(4):763-72.

11. Schwartz AE, Navedo AT, Berman MF. Pancuronium increases the duration of electroencephalogram burst suppression in dogs anesthetized with isoflurane. Anesthesiology. 1992;77(4):686-90.

12. Bock M, Klippel K, Nitsche B, Bach A, Martin E, Motsch J. Rocuronium potency and recovery characteristics during steady-state desflurane, sevoflurane, isoflurane or propofol anaesthesia. $\mathrm{Br} J$ Anaesth. 2000;84(1):43-7.

13. Itoh H, Shibata K, Matsumoto T, Nitta S, Nishi M, Kobayashi T, et al. Effects of neuromuscularblocking drugs in rats in vivo: direct measurements in the diaphragm and tibialis anterior muscle. Acta Anaesthesiol Scand. 2004;48(7):903-8.

14. Lanier WL, laizzo PA, Milde JH, Sharbrough FW. The cerebral and systemic effects of movement in response to a noxious stimulus in lightly anesthetized dogs. Possible modulation of cerebral function by muscle afferents. Anesthesiology. 1994;80(2):392-401.

15. Osawa T. Different recovery of the train-of-four ratio from rocuronium-induced neuromuscular blockade in the diaphragm and the tibialis anterior muscle in rat. J Anesth. 2008;22(3):236-41. 
16. Kurita T, Morita K, Kazama T, Sato S. Influence of cardiac output on plasma propofol concentrations during constant infusion in swine. Anesthesiology. 2002;96(6):1498-503.

17. Chemali JJ, Van Dort CJ, Brown EN, Solt K. Active emergence from propofol general anesthesia is induced by methylphenidate. Anesthesiology. 2012;116(5):998-1005.

18. Parks CL, Tucker W, Amlong CA, Mecozzi S, Pearce RA. Lipid-free fluoropolymer-based propofol emulsions and lipid reversal of propofol anesthesia in rats. Anesthesiology. 2016;124(6):1328-37.

19. Mansouri MT, Fidler JA, Meng QC, Eckenhoff RG, Garcia PS. Sex effects on behavioral markers of emergence from propofol and isoflurane anesthesia in rats. Behav Brain Res. 2019;367:59-67.

\section{Figures}

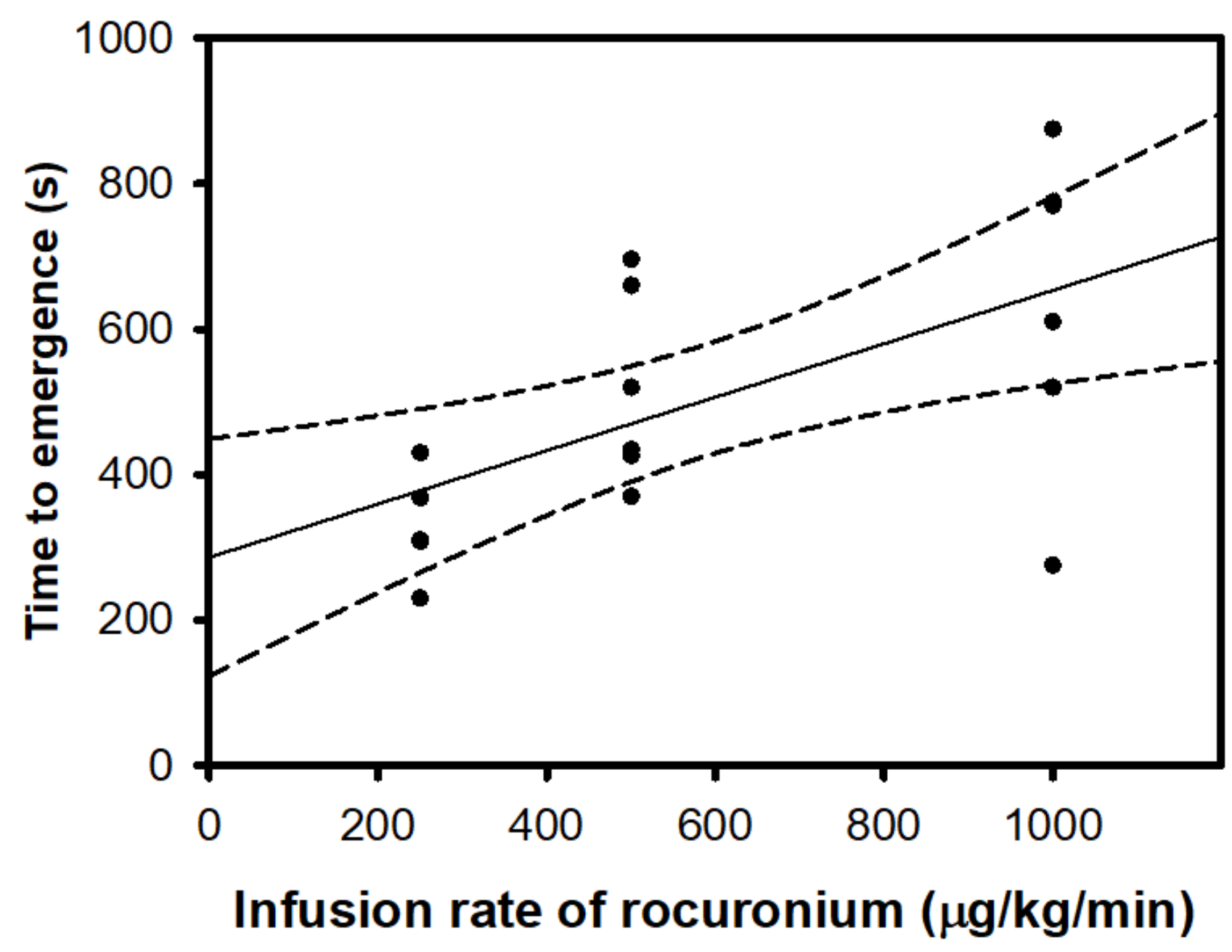

Figure 1

Relation between time to emergence from propofol anesthesia in rats and infusion rate of rocuronium along with propofol using a linear regression model (solid line) with corresponding $95 \%$ confidence 
intervals (dashed lines). Individual responses at each dose $(250,500,1000 \mu \mathrm{g} / \mathrm{kg} / \mathrm{min})$ are shown as black circles. The time to emergence from propofol anesthesia was dose-dependently prolonged by simultaneous infusion of rocuronium ( $\rho=0.624, p=0.006)$.

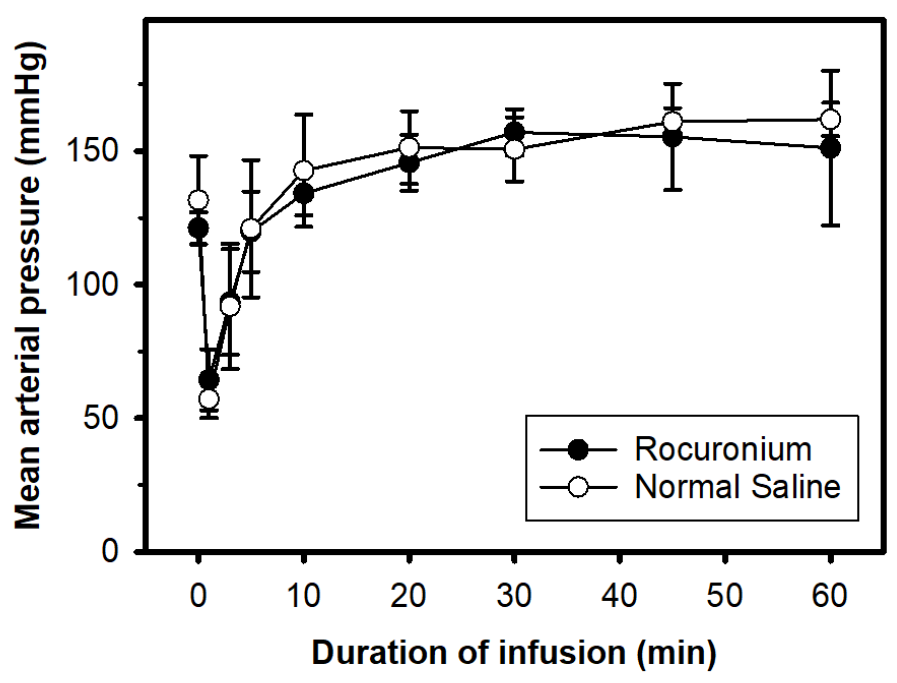

A

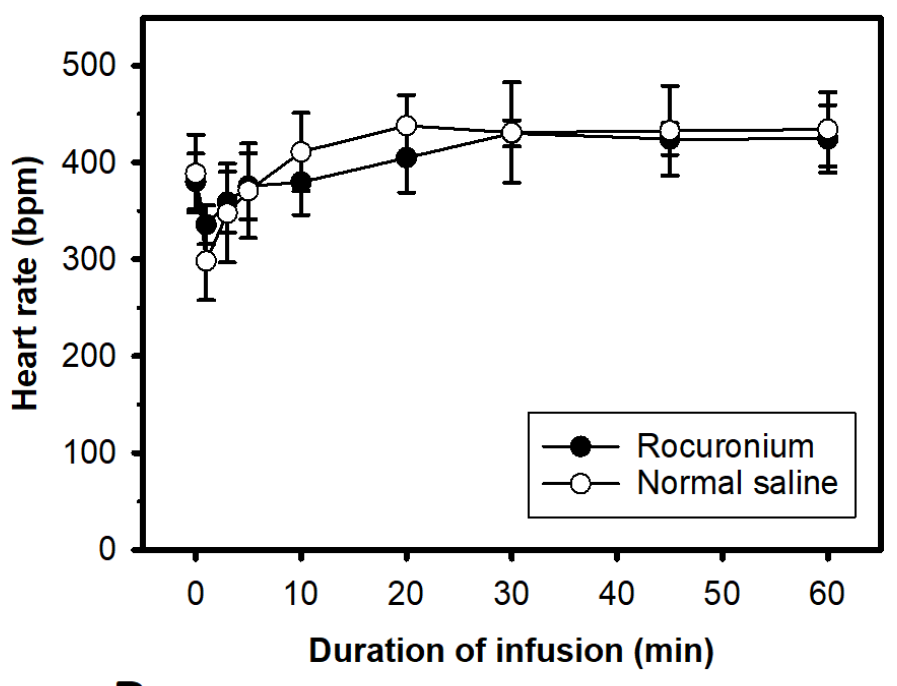

B

\section{Figure 2}

(a) Mean arterial pressure $(\mathrm{mmHg})$ measured at bolus injection of propofol (time 0 ) and at 1, 3, 5, 10, 20, 30,45 , and 60 min after initiating propofol infusion with rocuronium or normal saline. Mean arterial pressure after initiating propofol infusion with rocuronium did not differ significantly from that with normal saline at any time point. (b) Heart rate (beats per minute [bpm]) measured at bolus injection of propofol (time 0 ) and at 1, 3, 5, 10, 20,30, 45, and 60 min after initiating propofol infusion with rocuronium or normal saline. Heart rate after initiating propofol infusion with rocuronium did not differ significantly from that with normal saline at any time point.

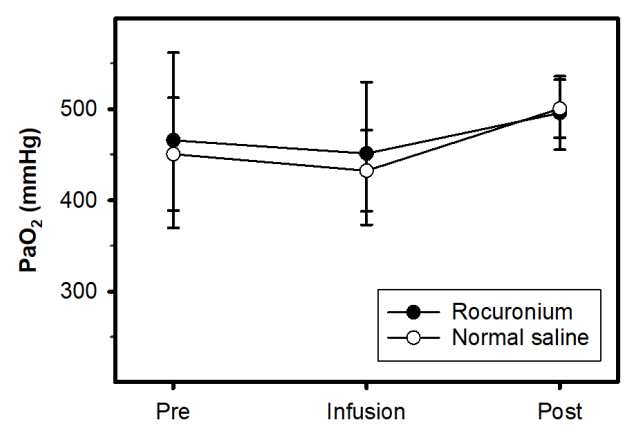

A

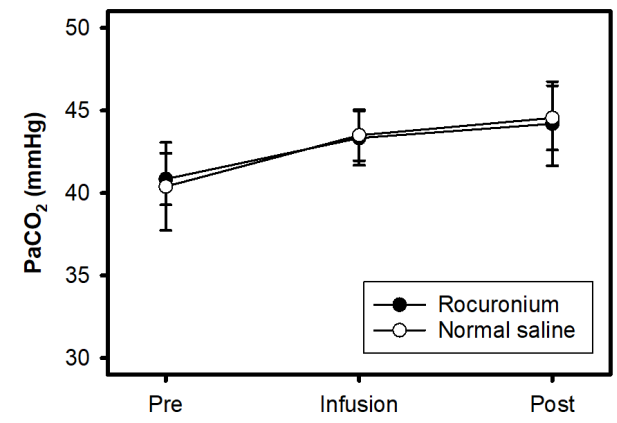

B

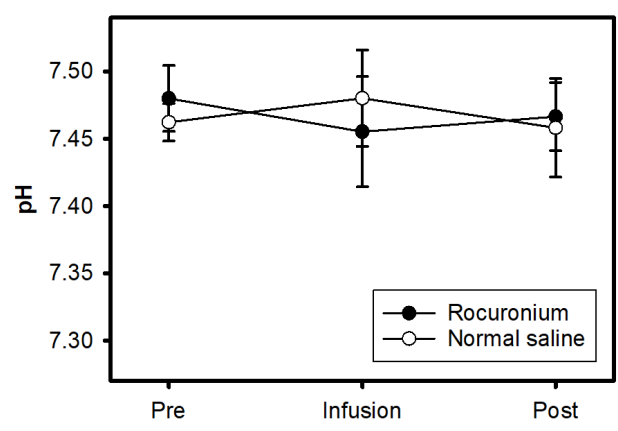

C

\section{Figure 3}

Blood gas analysis was performed at the following time points: before infusion (Pre); at 30 min after initiation of infusion (Infusion); and at 3 min after flushing the line at the discontinuation of infusion 
(Post). (a) partial pressure of oxygen (PaO2). (b) partial pressure of carbon dioxide (PaCO2). (c) pH. Values for $\mathrm{PaO} 2, \mathrm{PaCO} 2$, and $\mathrm{pH}$ did not differ significantly between the rocuronium group and the normal saline group at any time point.

\section{Supplementary Files}

This is a list of supplementary files associated with this preprint. Click to download.

- NC3RsARRIVEGuidelinesChecklist2014.docx 